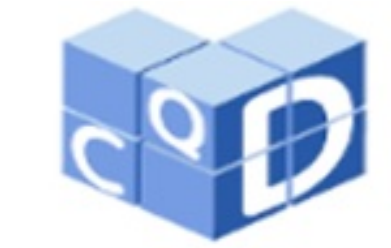

Revista Eletrônica Paulista de Matemática

ISSN 2316-9664

Volume 10, dez. 2017

Edição Ermac

Iniciação Científica

Beatriz Liara Carreira

UNESP - Universidade

Estadual Paulista "Júlio

de Mesquita Filho"

bia.liara36@hotmail.com

Leticia Braga Berlandi

UNESP - Universidade

Estadual Paulista "Júlio

de Mesquita Filho"

leticiaberlandi@gmail.com

Rafael de Lima Sterza

UNESP - Universidade

Estadual Paulista "Júlio

de Mesquita Filho"

rlsterza@gmail.com

Analice Costacurta Brandi

UNESP - Universidade

Estadual Paulista "Júlio

de Mesquita Filho"

analice@fct.unesp.br

\section{Solução numérica de problemas elípticos não lineares via método de diferenças finitas exponencial de alta ordem}

\author{
Numerical solution of nonlinear elliptic problems by the \\ exponential finite difference method of high order
}

\begin{abstract}
Resumo
Diversos fenômenos físicos são modelados por equações diferenciais parciais elípticas. Nem sempre essas equações admitem solução analítica, por isso a importância de buscar técnicas de soluções numéricas cada vez mais eficientes. O método de diferenças finitas é uma das técnicas numéricas mais antigas e populares na solução de equações diferenciais parciais. Recentemente, um novo método de diferenças finitas vem sendo estudado e aplicado na solução de equações elípticas lineares e não lineares. Trata-se do método de diferenças finitas exponencial de quarta ordem. A aplicação deste método em equações do segundo tipo faz surgir um sistema não linear que pode ser resolvido através de métodos iterativos, por exemplo, o método de Newton. Neste trabalho, uma equação elíptica não linear é resolvida através do método de diferenças finitas exponencial, e os resultados obtidos foram discutidos e comparados com resultados analíticos e numéricos presentes na literatura.
\end{abstract}

Palavras-chave: Equações não lineares. Diferenças finitas exponencial. Métodos numéricos e aplicações. Método de Newton.

\begin{abstract}
Several physical phenomena are modeled by elliptic partial differential equations. These equations do not always admit of an analytical solution, so the importance of searching for more efficient numerical solutions techniques. The finite difference method is one of the oldest and most popular numerical techniques in the solution of partial differential equations. Recently, a new finite difference method has been studied and applied in the solution of linear and nonlinear elliptic equations. This is the exponential finite difference method of the fourth order. The application of this method to equations of the second type gives rise to a nonlinear system that can be solved by iterative methods, for example Newton's method. In this work, a nonlinear elliptic equation is solved through the exponential finite difference method, and the results obtained were discussed and compared with analytical and numerical results in the literature.
\end{abstract}

Keywords: Nonlinear equations. Exponential finite difference. Numerical methods and applications. Newton's method. 


\section{Introdução}

Problemas de equilíbrio são aqueles em que a propriedade de interesse não depende da evolução temporal. Na maioria das vezes esses problemas são modelados por equações diferenciais parciais elípticas. Esses sistemas também são conhecidos como estacionários, justamente por permanecerem constantes em relação ao tempo.

As equações de Poisson e de Laplace, cujas aplicações são as mais variadas, são muito conhecidas. Por exemplo, a equação de Poisson representa o movimento de um fluido viscoso incompressível a baixa velocidade e a equação de Laplace é empregada para descrever potencial eletromagnético, dentre outras (FORTUNA, 2012), em que ambas representam fenômenos lineares. Entretanto a maior parte das equações que modelam os fenômenos físicos são caracterizadas pela sua não linearidade e lidar com equações desse tipo pode significar uma tarefa bastante complicada.

Essas equações podem ser resolvidas através de três tipos de abordagem: a computacional, a experimental e a analítica. Embora as duas últimas tenham suas vantagens, a abordagem computacional tem se sobressaído, pois não impõe limitações de ordem dimensional, física e espacial, tornando-a mais atrativa. Além disso, técnicas de estabilidade e convergência são incorporadas ao processo de solução garantindo a validade dos resultados.

A abordagem computacional se realiza através dos métodos numéricos que aproximam com determinada precisão a solução de equações diferenciais parciais através do auxílio de computadores. Recentemente, um novo método numérico foi desenvolvido por Pandey (PANDEY, 2013), chamado de método de diferenças finitas exponencial de quarta ordem e simulado computacionalmente para equações diferenciais parciais elípticas de segunda ordem, com diferentes condições de contorno.

Tratando-se de equações não lineares, o sistema resultante é também não linear e portanto é mais conveniente buscar sua solução através do método de Newton, que será tratado mais adiante. Neste contexto, o objetivo deste trabalho é resolver um problema não linear através do método exponencial em diferentes malhas comparando os resultados numéricos com resultados presentes na literatura, verificando o comportamento e eficiência do método na obtenção da solução.

\section{Formulação matemática}

O domínio de integração de uma equação diferencial parcial bidimensional é uma região $\Omega$ cuja área é limitada pela fronteira $\partial \Omega$.

$$
a(x, y) \frac{\partial^{2} u}{\partial x^{2}}+b(x, y) \frac{\partial^{2} u}{\partial x \partial y}+c(x, y) \frac{\partial^{2} u}{\partial y^{2}}=d\left(x, y, u, \frac{\partial u}{\partial x}, \frac{\partial u}{\partial y}\right) .
$$

A Equação ?? é considerada uma equação diferencial parcial elíptica em $\Omega$ se $b^{2}-4 a c<0$ para todo ponto interior de $\Omega$ (CUMINATO; MENEGUETTE JUNIOR, 2013).

Uma característica dos problemas modelados por equações diferenciais parciais elípticas é que toda região $\Omega$ seja imediatamente afetada por qualquer mudança no valor da variável dependente em um ponto do interior do domínio ou até mesmo na fronteira $\partial \Omega$. Isso é equivalente a dizer que as propriedades físicas de problemas elípticos propagam-se em todas as direções dentro do seu domínio e por este motivo suas condições de contorno normalmente são especificadas ao longo de toda fronteira. 
As condições de contorno usualmente especificam os valores da função ou os valores de sua derivada normal ao longo do contorno $\partial \Omega$, ou uma mistura de ambos, que são, respectivamente, as condições de Dirichlet ( $u$ é conhecida em $\partial \Omega$ ), de Neumann ( $\frac{\partial u}{\partial n}$ é conhecida em $\partial \Omega$ ) e Robin $\left(\alpha u=\beta \frac{\partial u}{\partial n}\right.$ conhecida em $\left.\partial \Omega\right)$. Em particular, considera-se neste trabalho uma região quadrada $\Omega=\{(x, y): a \leq x \leq b ; a \leq y \leq b\}$ com condição de contorno $u(x, y)=g(x, y)$ em $\partial \Omega$.

\section{Formulação numérica}

A resolução de uma equação diferencial parcial analiticamente fica restrita a problemas simples e lineares. Problemas complexos, como é o caso dos não lineares, tornam-se difíceis ou até mesmo impossíveis de se resolver analiticamente por conta da insuficiência dos métodos existentes. Enquanto isso, a abordagem computacional é capaz de solucionar equações com alto nível de complexidade, não havendo restrições a geometrias e processos complicados. É sempre necessário que haja um cuidado especial na construção da modelagem, para que de fato, ela seja uma representação do fenômeno em questão.

A abordagem computacional é realizada por meio dos métodos numéricos, que calculam soluções aproximadas para o problema através do suporte de computadores. Primeiramente, é necessário conhecer o domínio do problema, então alguns pontos são selecionados e nesses pontos aproxima-se a solução, uma vez que o computador seria incapaz de aplicar as técnicas numéricas num domínio contínuo, contendo infinitos pontos. A esse processo denomina-se discretização.

A discretização do domínio deve ser o primeiro passo para solucionar um problema estacionário através de uma equação elíptica. Para exemplificar o processo, considera-se $\Omega$ como sendo uma região quadrada, coberta por uma malha, conforme a Figura 1. Supondo o domínio adotado neste trabalho, então um ponto genérico $\left(x_{i}, y_{j}\right)$ desta malha é dado por $x_{i}=a+i h$, $i=1,2, \ldots, N$ e $y_{j}=a+j h, j=1,2, \ldots, N$, sendo $h=(b-a) / N$, com $N$ dado pelo número de subintervalos da malha.

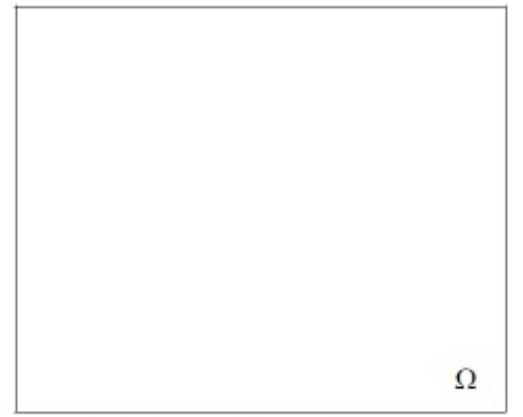

(a) Domínio contínuo.

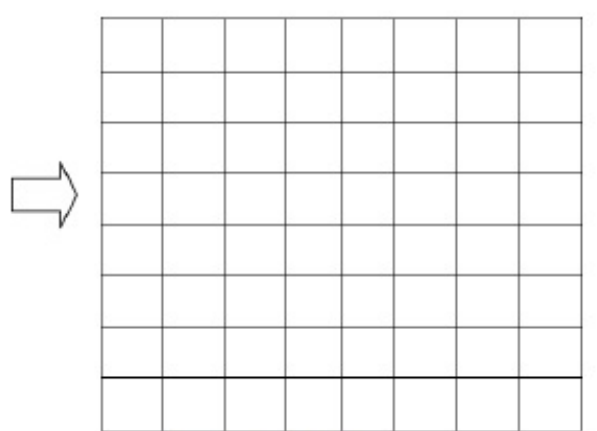

(b) Domínio discreto.

Figura 1: Discretização do domínio.

O método de diferenças finitas exponencial produz aproximações de alta ordem considerando um espaçamento uniforme $h$ nas direções $x$ e $y$. Inicialmente, é preciso considerar os oito pontos vizinhos $(i \pm 1, j),(i, j \pm 1)$ e $(i \pm 1, j \pm 1)$, ao ponto $(i, j)$ : 


$$
\left\{\begin{array}{l}
u_{i \pm 1, j}=u\left(x_{i} \pm h, y_{j}\right) \\
u_{i, j \pm 1}=u\left(x_{i}, y_{j} \pm h\right) \\
u_{i \pm 1, j \pm 1}=u\left(x_{i} \pm h, y_{j} \pm h\right)
\end{array}\right.
$$

com $i=0,1, \ldots, N$, sendo $N$ a quantidade de subintervalos tomados.

Para aproximar a solução analítica da equação não linear:

$$
\frac{\partial^{2} u}{\partial x^{2}}+\frac{\partial^{2} u}{\partial y^{2}}=f(x, y, u)
$$

o método de diferenças finitas exponencial utiliza nove pontos e inicialmente é dado pela seguinte equação:

$$
a_{0}\left(u_{i+1, j}+u_{i-1, j}+u_{i, j+1}+u_{i, j-1}\right)+a_{1}\left(u_{i+1, j+1}+u_{i-1, j+1}+u_{i+1, j-1}+u_{i-1, j-1}\right)+a_{2} u_{i, j}=b_{0} h^{2} f_{i, j} e^{\phi(h)},
$$

onde $a_{0}, a_{1}, a_{2}$ e $b_{0}$ são constantes e $\phi(h)$ é uma função diferenciável. A partir dessa equação, pode-se ainda, definir a função $F(h, u)$ da seguinte forma:

$$
\begin{aligned}
F(h, u) \equiv & a_{0}\left(u_{i+1, j}+u_{i-1, j}+u_{i, j+1}+u_{i, j-1}\right)+ \\
& +a_{1}\left(u_{i+1, j+1}+u_{i-1, j+1}+u_{i+1, j-1}+u_{i-1, j-1}\right)+a_{2} u_{i, j}-b_{0} h^{2} f_{i, j} e^{\phi(h)}=0 .
\end{aligned}
$$

Observando a Figura ??, nota-se a disposição dos pontos envolvidos na expressão de discretização de uma equação diferencial utilizando o método de diferenças finitas exponencial.

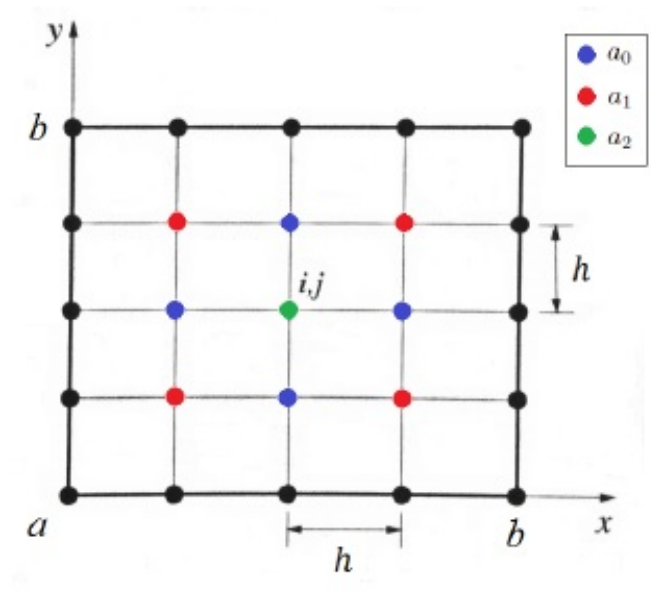

Figura 1: Estêncil da aproximação pelo método de diferenças finitas exponencial.

A partir da Equação ?? ocorre o desenvolvimento do método de diferenças finitas exponencial de alta ordem. Primeiramente, expandindo a função diferenciável $\phi(h)$ em série de Mac Lauren obtém-se a seguinte expressão (CARREIRA et al., 2017):

$$
\phi(h)=\phi(0)+h \phi^{\prime}(0)+\frac{h^{2}}{2 !} \phi^{\prime \prime}(0)+O\left(h^{3}\right) .
$$


Agora, aplicando expansão exponencial no termo $e^{\phi(h)}$, o resultado será:

$$
e^{\phi(h)}=1+\phi(h)+\frac{\phi(h)^{2}}{2 !}+O\left(h^{3}\right) .
$$

Então, o desenvolvimento do método exige que se faça a substituição da Equação ?? na Equação ??. Esse processo acaba fornecendo a expressão a seguir:

$$
e^{\phi(h)}=1+\phi(0)+\frac{1}{2} \phi(0)^{2}+h(1+\phi(0)) \phi^{\prime}(0)+\frac{h^{2}}{2}\left(\phi^{\prime}(0)^{2}+(1+\phi(0)) \phi^{\prime \prime}(0)\right)+O\left(h^{3}\right) .
$$

Dando continuidade ao processo, é preciso expandir $F(h, u)$ em série de Taylor em torno do ponto $u_{i, j}$. Truncando essa expansão até a quarta ordem, pode-se escrevê-la da seguinte maneira:

$$
\begin{aligned}
F_{i, j}= & a_{0} 4 u_{i, j}+a_{1} 4 u_{i, j}+a_{2} u_{i, j}-b_{0} h^{2} f_{i, j} e^{\phi(h)}+ \\
& +a_{0}\left(h^{2} \frac{\partial^{2} u}{\partial x^{2}}+h^{2} \frac{\partial^{2} u}{\partial y^{2}}\right)_{(i, j)}+a_{1}\left(2 h^{2} \frac{\partial^{2} u}{\partial x^{2}}+2 h^{2} \frac{\partial^{2} u}{\partial y^{2}}\right)_{(i, j)}+ \\
& +a_{0}\left(\frac{h^{4}}{12} \frac{\partial^{4} u}{\partial x^{4}}+\frac{h^{4}}{12} \frac{\partial^{4} u}{\partial y^{4}}\right)_{(i, j)}+a_{1}\left(\frac{h^{4}}{6} \frac{\partial^{4} u}{\partial x^{4}}+\frac{h^{4}}{6} \frac{\partial^{4} u}{\partial y^{4}}+h^{4} \frac{\partial^{4} u}{\partial x^{2} \partial y^{2}}\right)_{(i, j)} .
\end{aligned}
$$

Para simplificar a expressão será preciso considerar $f_{i, j}=\left(\frac{\partial^{2} u}{\partial x^{2}}+\frac{\partial^{2} u}{\partial y^{2}}\right)_{(i, j)}$, e então:

$$
\nabla^{2} f_{i, j}=\left(\frac{\partial^{2} u}{\partial x^{2}}+\frac{\partial^{2} u}{\partial y^{2}}\right)\left(\frac{\partial^{2} u}{\partial x^{2}}+\frac{\partial^{2} u}{\partial y^{2}}\right)_{(i, j)} .
$$

Usando essas notações e substituindo-as na Equação ??, tem-se:

$$
\begin{array}{r}
\left(4 a_{0}+4 a_{1}+a_{2}\right) u_{i, j}+\left(a_{0}+2 a_{1}\right) h^{2} f_{i, j}+\frac{h^{4}}{12}\left(a_{0}+2 a_{1}\right) \nabla^{2} f_{i, j}+ \\
-\frac{h^{4}}{6}\left(a_{0}-4 a_{1}\right) \frac{\partial^{4} u}{\partial x^{2} \partial y^{2}}-b_{0} h^{2} f_{i, j}\left(1+\phi(0)+\frac{1}{2} \phi(0)^{2}+\right. \\
\left.+h(1+\phi(0)) \phi^{\prime}(0)+\frac{h^{2}}{2}\left(\phi^{\prime}(0)^{2}+(1+\phi(0)) \phi^{\prime \prime}(0)\right)\right)=0 .
\end{array}
$$

Analisando os coeficientes de $h^{p}, \operatorname{com} p=0,2,3,4$, constrói-se o seguinte sistema não linear:

$$
\left\{\begin{array}{l}
4 a_{0}+4 a_{1}+a_{2}=0 \\
\left(a_{0}+2 a_{1}\right) f_{i, j}-b_{0} f_{i, j}\left(1+\phi(0)+\frac{1}{2} \phi(0)^{2}\right)=0 \\
-b_{0} f_{i, j}(1+\phi(0)) \phi^{\prime}(0)=0 \\
\lambda_{1}-\lambda_{2}-\lambda_{3}=0
\end{array}\right.
$$

sendo:

$$
\begin{aligned}
& \lambda_{1}=\frac{1}{12}\left(a_{0}+2 a_{1}\right) \nabla^{2} f_{i, j}, \\
& \lambda_{2}=\frac{1}{6}\left(a_{0}-4 a_{1}\right)\left(\frac{\partial^{4} u}{\partial x^{2} \partial y^{2}}\right)_{(i, j)}, \\
& \lambda_{3}=b_{0} f_{i, j} \frac{1}{2}\left(\phi^{\prime}(0)^{2}+(1+\phi(0)) \phi^{\prime \prime}(0)\right) .
\end{aligned}
$$


Para resolver o sistema anterior, considera-se $\phi(0)=0, \phi^{\prime}(0)=0$ e $a_{0}-4 a_{1}=0$. Com simples substituições convenientes descobre-se facilmente os valores de $a_{0}, a_{2}$ e $b_{0}$, como segue a partir de agora:

$$
\begin{aligned}
& a_{0}=4 a_{1}, \\
& a_{2}=-20 a_{1}, \\
& b_{0}=6 a_{1} .
\end{aligned}
$$

A substituição de todos esses valores conhecidos na última equação do sistema não linear fornece o valor de $\phi^{\prime \prime}(0)$ :

$$
\phi^{\prime \prime}(0)=\frac{\nabla^{2} f_{i, j}}{6 f_{i, j}} .
$$

Agora que são conhecidos, os valores de $\phi(0), \phi^{\prime}(0)$ e $\phi^{\prime \prime}(0)$ são utilizados na Equação ??, obtendo-se o valor de $\phi(h)$ :

$$
\phi(h)=\frac{h^{2} \nabla^{2} f_{i, j}}{12 f_{i, j}} .
$$

Finalmente, sabe-se os valores de todas as constantes que a princípio eram desconhecidas e além disso, uma expressão foi encontrada para a função $\phi$. Substituindo os valores de $a_{0}, a_{2}, b_{0}$ e $\phi(h)$ na Equação ??, obtém-se a equação do método de diferenças finitas exponencial na sua forma final:

$$
4\left(u_{i+1, j}+u_{i-1, j}+u_{i, j+1}+u_{i, j-1}\right)+\left(u_{i+1, j+1}+u_{i-1, j+1}+u_{i+1, j-1}+u_{i-1, j-1}\right)-20 u_{i, j}=6 h^{2} f_{i, j} e^{\frac{h^{2} \nabla^{2} f_{i, j}}{12 f_{i, j}}} .
$$

O laplaciano que se encontra no expoente é aproximado por diferenças finitas de segunda ordem, como visto a seguir:

$$
h^{2} \nabla^{2} f_{i, j}=f_{i+1, j}+f_{i-1, j}-4 f_{i, j}+f_{i, j+1}+f_{i, j-1} .
$$

Essa expressão é a discretização da equação diferencial utilizando o método de diferenças finitas, também conhecida pela fórmula dos cinco pontos.

Tratando-se de uma equação diferencial parcial elíptica não linear, a discretização dessa equação pelo método de diferenças finitas exponencial fornecerá um sistema não linear que deverá ser resolvido por um método iterativo. Neste trabalho foi utilizado o método de Newton.

Suponha um sistema de $n$ equações não lineares a $n$ incógnitas:

$$
\left\{\begin{array}{c}
f_{1}\left(x_{1}, x_{2}, \ldots, x_{n}\right)=0 \\
f_{2}\left(x_{1}, x_{2}, \ldots, x_{n}\right)=0 \\
\vdots \\
f_{n}\left(x_{1}, x_{2}, \ldots, x_{n}\right)=0
\end{array}\right.
$$

Com a definição dos vetores $x=\left[x_{1}, x_{2}, \ldots, x_{n}\right]^{T}$ e $f=\left[f_{1}, f_{2}, \ldots, f_{n}\right]^{T}$, pode-se escrever o sistema não linear na sua forma compacta:

$$
f(x)=0
$$


O método de Newton para aproximar raízes de uma função escalar $f$ é dado por:

$$
x^{(k+1)}=x^{(k)}-\frac{f\left(x^{(k)}\right)}{f^{\prime}\left(x^{(k)}\right)}, \quad k \geq 0,
$$

considerando $k$ a quantidade de iterações e portanto sendo $x^{(0)}$ uma aproximação inicial da solução de $f$.

Para aplicar este método de Newton à resolução de um sistema não linear, substitui-se a primeira derivada da função escalar $f$ pela matriz jacobiana da função vetorial $f$ (QUARTERONI; SALERI, 2006):

$$
J=\left(\begin{array}{cccc}
\frac{\partial f_{1}}{\partial x_{1}} & \frac{\partial f_{1}}{\partial x_{2}} & \cdots & \frac{\partial f_{1}}{\partial x_{n}} \\
\frac{\partial f_{2}}{\partial x_{1}} & \frac{\partial f_{2}}{\partial x_{2}} & \cdots & \frac{\partial f_{2}}{\partial x_{n}} \\
\vdots & \vdots & \ddots & \vdots \\
\frac{\partial f_{n}}{\partial x_{1}} & \frac{\partial f_{n}}{\partial x_{2}} & \cdots & \frac{\partial f_{n}}{\partial x_{n}}
\end{array}\right),
$$

em que o símbolo $\frac{\partial f_{i}}{\partial x_{j}}$, com $i, j=1,2, \ldots, n$ representa a derivada parcial de $f_{i}$ em relação a $x_{j}$.

Logo, o método se resume em:

$$
x^{(k+1)}=x^{(k)}+\Delta x^{(k)},
$$

sendo $J \Delta x^{(k)}=-f\left(x^{(k)}\right)$ e $x^{(0)}$ um vetor inicial escolhido previamente de maneira conveniente, isto é, este vetor deve estar próximo da solução analítica para garantir que a solução do método iterativo convirja para a solução do sistema.

Neste trabalho, o código computacional e também os testes numéricos foram implementados utilizando o software Matlab.

\section{Resultados numéricos}

Para análise dos resultados provenientes da aplicação dos métodos numéricos tratados nas seções anteriores, será utilizada a seguinte equação elíptica não linear:

$$
\frac{\partial^{2} u}{\partial x^{2}}+\frac{\partial^{2} u}{\partial y^{2}}=-f(x, y, u)
$$

$\operatorname{com} f(x, y, u)=\frac{\pi^{2}}{4} u(1-u)+2 \operatorname{sen}\left(\frac{\pi y}{2}\right)+\frac{\pi^{2}}{4}\left(1-x^{2}\right)^{2} \operatorname{sen}^{2}\left(\frac{\pi y}{2}\right)$ (HU; CHEN, 2007).

A Equação ?? é definida num domínio quadrado com $0 \leq x, y \leq 1$ e condição de contorno do tipo Dirichlet definido por $u(x, y)$, sendo $u$ a solução analítica dada por $u(x, y)=\left(1-x^{2}\right) \operatorname{sen}\left(\frac{\pi}{2} y\right)$. Após a discretização pelo método exponencial, o sistema não linear resultante será resolvido pelo método de Newton, considerando um vetor solução inicial $x^{(0)}=[0,0, \ldots, 0]^{T}$.

As soluções obtidas nas simulações referentes a este problema são comparadas com a solução presente no artigo de Pandey (PANDEY, 2013). Serão utilizados como comparação o erro máximo absoluto (MAU) e a quantidade de iterações realizadas em cada simulação. Além disso, o problema será simulado em uma malha grossa e uma malha fina, $\operatorname{com} N=4$ e $N=8$, respectivamente.

$$
M A U=\max \left|x^{(k)}-x\right|
$$


Tabela 1: Resultados obtidos por Pandey (PANDEY, 2013).

\begin{tabular}{|c|c|c|}
\hline Malha & MAU & Iterações \\
\hline Grossa & $2.8193 \mathrm{e}-05$ & 11 \\
Fina & $1.7285 \mathrm{e}-06$ & 22 \\
\hline
\end{tabular}

Tabela 2: Resultados obtidos neste trabalho.

\begin{tabular}{|c|c|c|c|}
\hline Malha & MAU & Iterações & Tempo Computacional \\
\hline Grossa & $2.8150 \mathrm{e}-05$ & 4 & $6.4817 \mathrm{~s}$ \\
Fina & $1.8190 \mathrm{e}-06$ & 4 & $120.2692 \mathrm{~s}$ \\
\hline
\end{tabular}

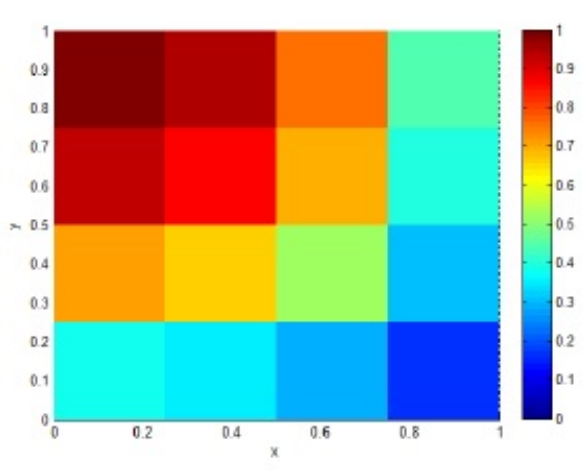

(a) Solução numérica.

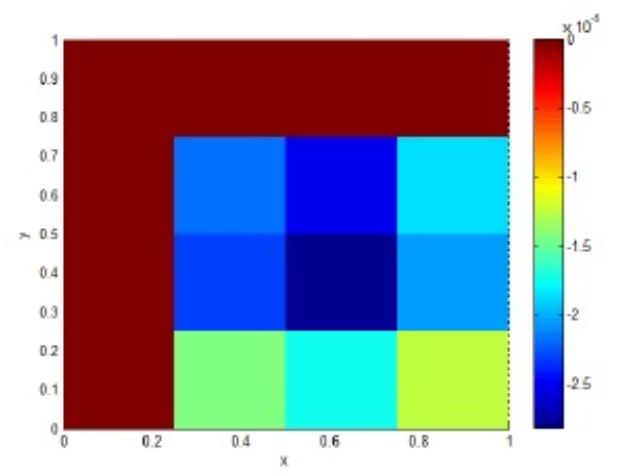

(b) Erro.

Figura 3: Resultados referentes à malha grossa.

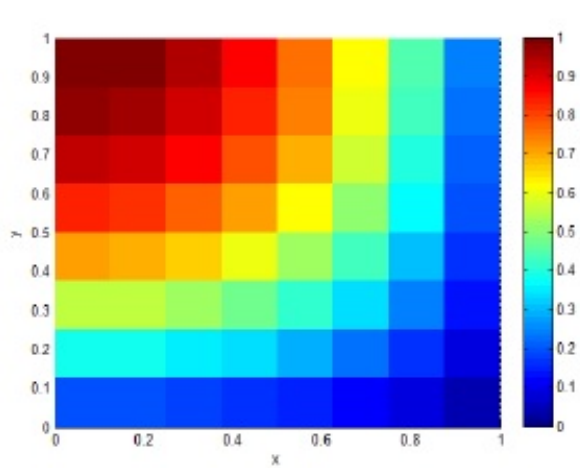

(a) Solução numérica.

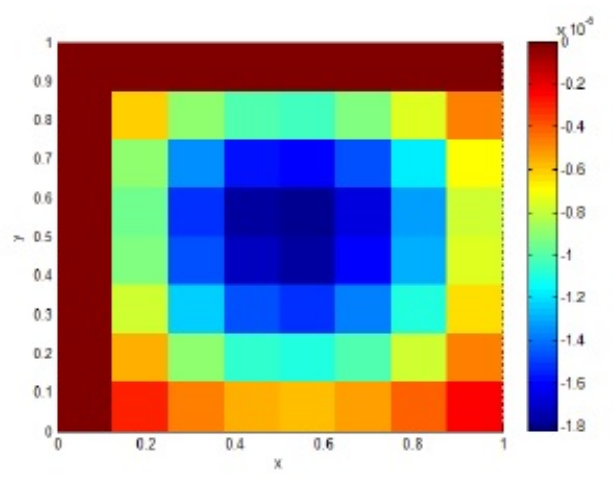

(b) Erro.

Figura 4: Resultados referentes à malha fina.

É possível notar que as aproximações obtidas no presente trabalho e por Pandey (PANDEY, 2013) foram bem semelhantes, mantendo a mesma ordem de erro para cada malha adotada. A principal diferença está na quantidade de iterações realizadas em cada teste. Esta ocorrência pode estar relacionada com o critério de parada adotado ou mais provavelmente com o vetor solução inicial considerado pelo autor. Verifica-se ainda que o tempo computacional gasto nas simulações realizadas no presente estudo foi relativamente pequeno, considerando-se tratar de problemas que envolvem a resolução de sistemas não lineares, o que é sempre mais complicado do ponto de vista 
numérico e computacional.

\section{Conclusão}

O método exponencial é uma nova técnica de diferenças finitas que tem apresentado bons resultados, e que responde bem ao refinamento da malha, calculando melhores aproximações em malhas mais finas, cujo espaçamento foi reduzido. De modo geral, este método apresentou um bom desempenho em problemas elípticos não lineares. Com a aproximação inicial adotada o método foi capaz de convergir após realizar poucas iterações. Com base nos resultados gerados até aqui, as aproximações acompanham um erro da ordem de $10^{-5}$ e $10^{-6}$, o que representa uma boa aproximação. Isso mostra o quanto este método pode ser considerado competitivo quando comparado a métodos mais clássicos.

\section{Agradecimentos}

Os autores agradecem à FAPESP pelo apoio financeiro no desenvolvimento deste trabalho, processo $n^{0} 2016 / 08343-2$.

\section{Referências bibliográficas}

CARREIRA, B. L. et al. Solução numérica de problemas elípticos não lineares via método de diferenças finitas exponencial de alta ordem. In: ENCONTRO REGIONAL DE MATEMÁTICA APLICADA E COMPUTACIONAL, 4., 2017, Bauru. Caderno de trabalhos completos e resumos. Bauru: Unesp, Faculdade de Ciências, 2017. p. 366-373. Disponível em: 〈http://www.fc.unesp.br/ $\ \# ! \backslash \backslash$ departamentos/matematica/eventos2341/ermac/ cadesnos-de-trabalhos-completos-e-resumos/>. Acesso em: 03 out. 2017.

CUMINATO, J. A.; MENEGUETTE JUNIOR, M. Discretização de equações diferenciais parciais: técnicas de diferenças finitas. Rio de Janeiro: SBM, 2013.

FORTUNA, A. O. Técnicas computacionais para dinâmica dos fluidos: conceitos básicos e aplicações. 2. ed. São Paulo: EDUSP, 2012.

HU, H. Y.; CHEN, J. S. Radial basis collocation method and quasi-Newton iteration for nonlinear elliptic problems. Numerical Methods for Partial Differential Equations, v. 24, n. 3, p. 991-1017, 2007.

PANDEY, P. K. A higher accuracy exponential finite difference method for the numerical solution of second order elliptic partial differential equations. Journal of Mathematical and Computational Science, v. 3, n. 5, p. 1325-1334, 2013.

QUARTERONI, A.; SALERI, F. Cálculo científico com MATLAB e Octave. 2. ed. Milano: Springer-Verlag, 2006.

Artigo recebido em jun. 2017 e aceito em nov. 2017.

CARREIRA, B. L. et al. Solução numérica de problemas elípticos não lineares via método de diferenças finitas exponencial de alta ordem. C.Q.D.- Revista 